\title{
The VIV detection based on Poincare surface of sections
}

\author{
Xingfu Zhong, Liming Lin, Yingxiang $\mathrm{Wu}$ \\ Key Laboratory for Hydrodynamics and Ocean Engineering \\ Institute of Mechanics, CAS \\ Beijing, China \\ xfzhong@imech.ac.cn
}

\author{
Yang He, Peilin Liu, Xiaodong Pan \\ Engineering Company \\ Offshore Oil Engineering Co. Ltd. \\ Tianjin, China \\ heyang@mail.cooec.com.cn
}

\begin{abstract}
By Poincare surface of sections, dynamical features of manifold in two, three or high dimensions can be investigated. Characteristics of Poincare sections for all kinds of manifold can be obtained qualitatively, which is used to study system with high dimensions by reducing dimension. In present work, a method of quick detection on occurrence of vortex-induced vibrations (VIV) of cylindrical structure is developed from measured time history of one variable by using Poincare surface of section. Experimental results have shown that such method is quick and reliable as a new means of engineering detection on VIV of cylindrical structure.
\end{abstract}

Keywords-Poincare surface of sections; detection; vortexinduced vibraion

\section{INTRODUCTION}

In oil-gas production system in deep sea, flexible risers are key instruments connecting production platform to subsea drill and production equipments. When the ocean flow passes around a riser, vortex formed behind both sides of body is alternately shed. The periodic and fluctuating fluid force on surfaces of structure is thus generated, especially in vertical direction which is orthogonal to the flow direction. As a result of it, the vibration of body is induced, called vortex-induced vibrations. If the frequency of vortex shedding is close or equal to the natural frequency of structure, the lock-in or synchronization is occurred. Meantime, the amplitude of structural oscillation is increased greatly. This leads to the instability and fatigue damage of risers in deep sea as a main reason. In a few decades, shutdown of oil-gas production and consequently oil pollution has been frequently occurred due to great damage of risers under VIV, which not only results in huge economical costs, but also dangers marine environment. Therefore, the safety of risers is widely focused on in ocean engineering, which is also highlighted as one of key interests for academe and engineer. Further information and knowledge of VIV can be referenced in reviews $[1,2,3]$.

\section{BASIC PRINCIPLE}

\section{A. Poincare section}

Dynamic system is varied with time, so its status is. Hence, the status space is needed to describe it. Such space is often composed of a kind of manifold multiplied with space of time coordinate. This manifold is a curved space including the flat plane space to indicate all possible situations in system for configuration at certain time, called configuration space. Dynamic system is a kind of mapping from the status space of model to configuration space. Then, a dynamical system is a mapping $[4,5]$,

$$
\vec{\Phi}: \vec{M} \times \vec{R} \rightarrow \vec{M}
$$

where the vector of $M$ is a manifold, which could be an Euclidean space as a flat plane space, $R^{n}$, or a curved space, such as a curved line, a curved plane, or a super-curved plane.

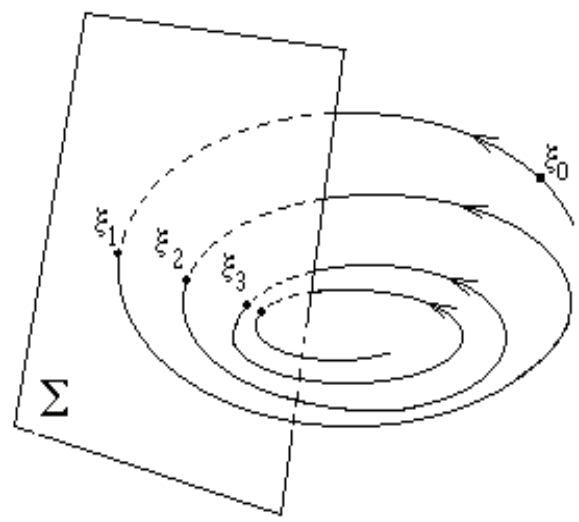

Figure1. Schematic diagram of Poincare section.

In a n-dimensional space, the (n-1)-dimensional supercurved plane $\Sigma$ is taken from the manifold, as shown in Fig.1, where $\xi_{0}$ is a point in the n-dimensional space. The trajectory path through $\xi_{0}$ intersects with $\Sigma$ at the point $\xi_{1}$. Then, a series of intersectional points, $\xi_{2}, \xi_{3}$, and so on, would be obtained. Relationship of these points can be explained as $\xi_{n+1}=\mathrm{P}\left(\xi_{n}\right)$, where $\mathrm{P}$ is called Poincare surface of section. The investigation on $\mathrm{P}$ is simple because $\mathrm{P}$ is the intersection in the (n-1)dimensional space. By Poincare section, dynamic behaviors of manifold in two, three or high dimensional space could be analyzed. Features of Poincare section could be also obtained for manifolds qualitatively. High-dimensional system can be investigated through number of dimensions lowered by Poincare section, as expressed as following, 


$$
x_{n+1}=f_{\left(x_{n}\right)}
$$

\section{B. Measurement of signals}

In process of measurement, signals in engineering, $x_{(t)}$, can be defined as,

$$
x_{(t)}=x_{p(t)}+x_{n(t)}+n_{(t)},
$$

where $x_{p(t)}$ is a periodic component equal to $x_{p(t+T)}$, and $T$ is a fundamental period, $x_{n(t)}=g_{(t)} \cdot x_{r(t)}$ is an aperiodic component, $g_{(t)}$ is also a periodic function, $x_{r(t)}$ is a random function, $n_{(t)}$ is a noise with a random interference.

Therefore, a treatment in statistics is needed to be carried out in an analysis of signals. The estimation of real signals is then well obtained. The process of such treatment can be simplified by Poincare surface of sections. The basic characteristics of measured signals in engineering for dynamic behaviors in $\mathrm{n}$-dimensional space are achieved in (n-1)dimensional space.

\section{EXPERIMENTAL INTRODUCTION}

In present experiments, the fluid load on structure is represented by the extent of oscillating structure. Such oscillation is illustrated by the pendulum motion, rather than the translation motion. The oscillating amplitude of structural VIV is measured to express the extent of fluid loads. When the frequency of vortex shedding is close to the natural frequency of pendulum, the synchronization is occurred with the amplitude reaching up to the maximum. The velocity of incoming flow is then estimated for identifying the specific situation of synchronization of VIV of structure.

Experiments were carried out in the circulation water channel at the Key Laboratory for Hydrodynamics and Ocean Engineering, Institute of Mechanics, Chinese Academy of Sciences. As shown in Fig.2, the channel was the depth of $1 \mathrm{~m}$. The maximum velocity reached up to $1 \mathrm{~m} / \mathrm{s}$. The test section was $1 \mathrm{~m}$ wide, $6 \mathrm{~m}$ long, and 0.65 to $0.7 \mathrm{~m}$ deep.

\section{ANALYSIS OF MEASURED SIGNALS OF VIV}

Major measured data include four aspects or steps. The first is the natural frequency of test cylinder oscillating freely in air and still water, which can predict the incoming velocity when synchronization is occurred. The second is the real velocity of incoming flow. The third is the measurement of oscillating amplitudes of a specific test cylinder at different velocities, 2, $5,8,10,13,15,18$ and $20 \mathrm{~cm} / \mathrm{s}$. The last is the repeated third step with different cylinder. When the varied velocity becomes stable, measured signals are recorded by computation. As shown in Fig.3, for different shapes of cylinders, $0^{\#}$ is a straight circular cylinder, $1^{\#}, 2^{\#}$ and $3^{\#}$ are harmonic cylinder with a circular section, and $4^{\#}, 5^{\#}$ and $6^{\#}$ are cone-like cylinder.

Figures 4 and Figures 5 are time histories and frequency analysis for vertical VIV of $0^{\#}$ and $5^{\#}$. In figures of time histories, horizontal coordinate is the measured time $\left(\mathrm{s}^{*} 1 / 10\right)$ and vertical coordinate non-dimensional amplitude of oscillation (ratio of amplitude d to diameter of cylinder D). In figures of frequency analysis, horizontal coordinate is frequency $(\mathrm{Hz})$ and vertical coordinate power spectral density (PSD).

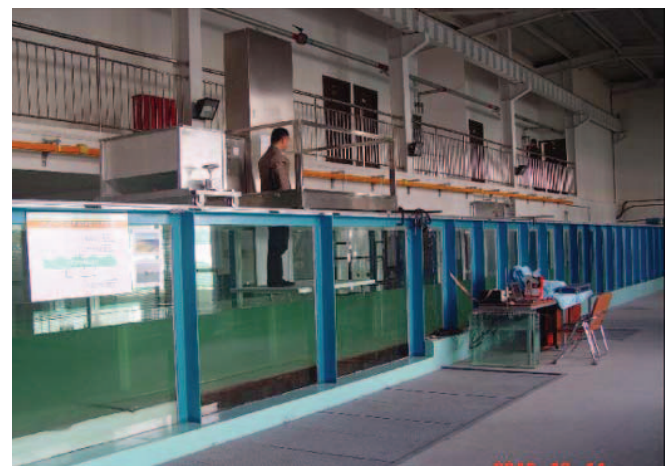

(a)

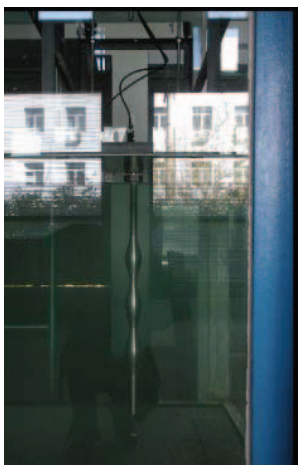

(b)

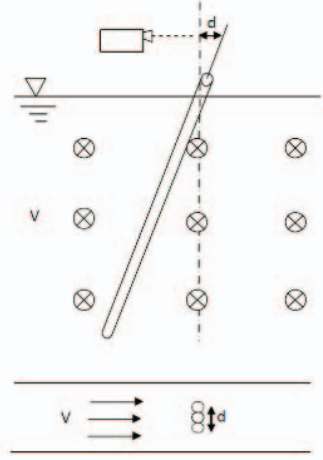

(c)
Figure 2. Experimental instruments, (a) circulating water channel, (b) text section, (c) schematic of oscillating measurement.
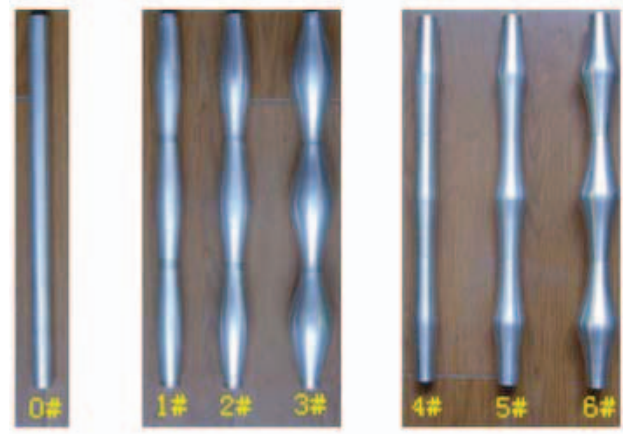

Figure 3. Different shapes of cylindrical structure $\left(0^{\#}\right.$ is straight, $1^{\#}, 2^{\#}$ and $3^{\#}$ are hamony, $4^{\#}, 5^{\#}$ and $6^{\#}$ are cone-like).

\section{QUICK DETECTIONS OF STRUCTURAL VIV}

The major aim in detection of parameters is to obtain useful information of measured signals. The key is focused on the extraction of features from the time history of signals, which are related with the velocity of fluid, oscillating amplitude and frequency. By applying Poincare surface of sections in time histories of non-dimensional oscillating amplitude, the representative characteristic patterns can be obtained. From time histories and Poincare sections, some results have been analyzed as following, 


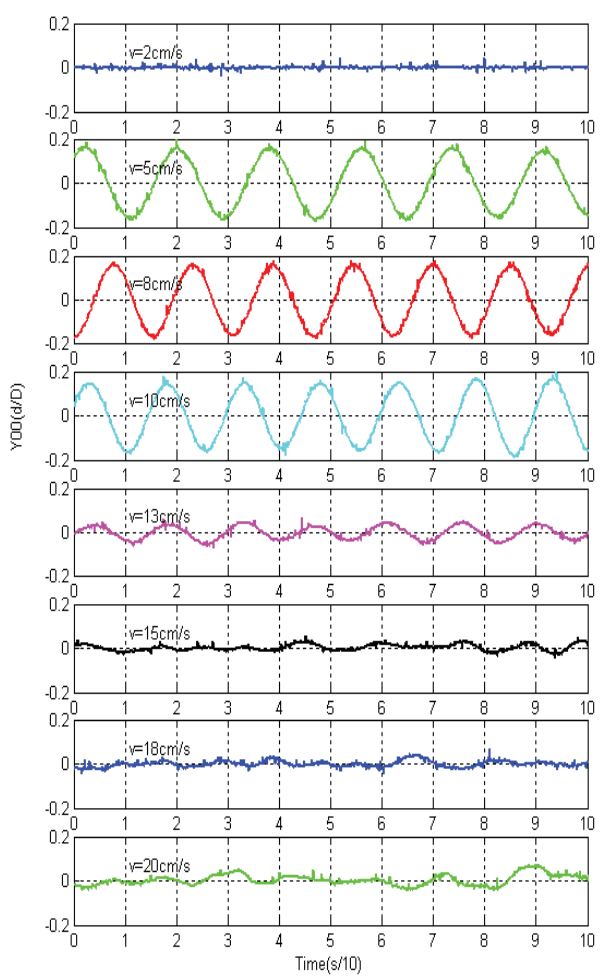

(a)

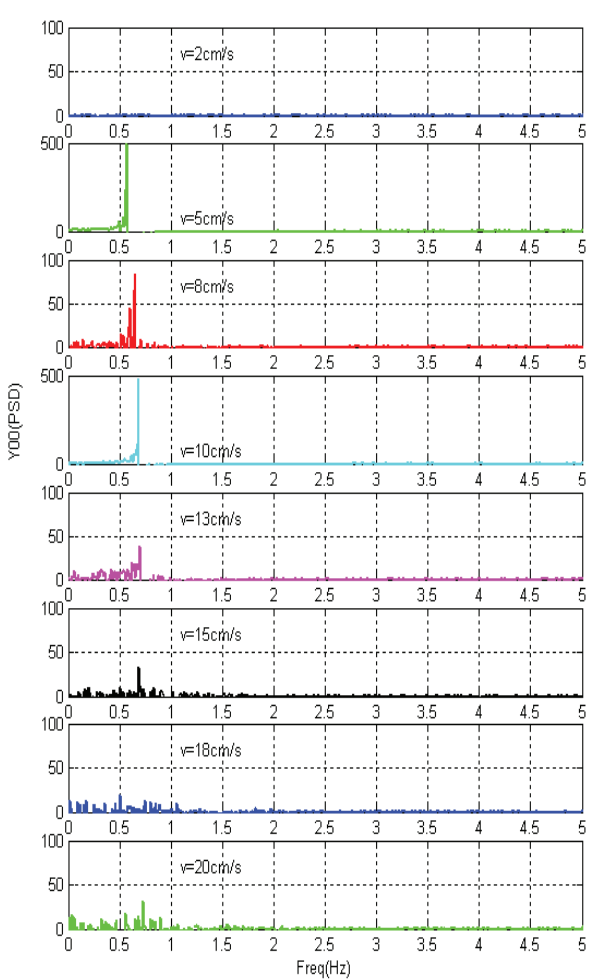

(b)

Figure 4. (a) Time histories of non-dimensional amplitudes of vertical oscillation and (b) their power spectral density (PSD) for $0^{\#}$ cylinder at different velocities.
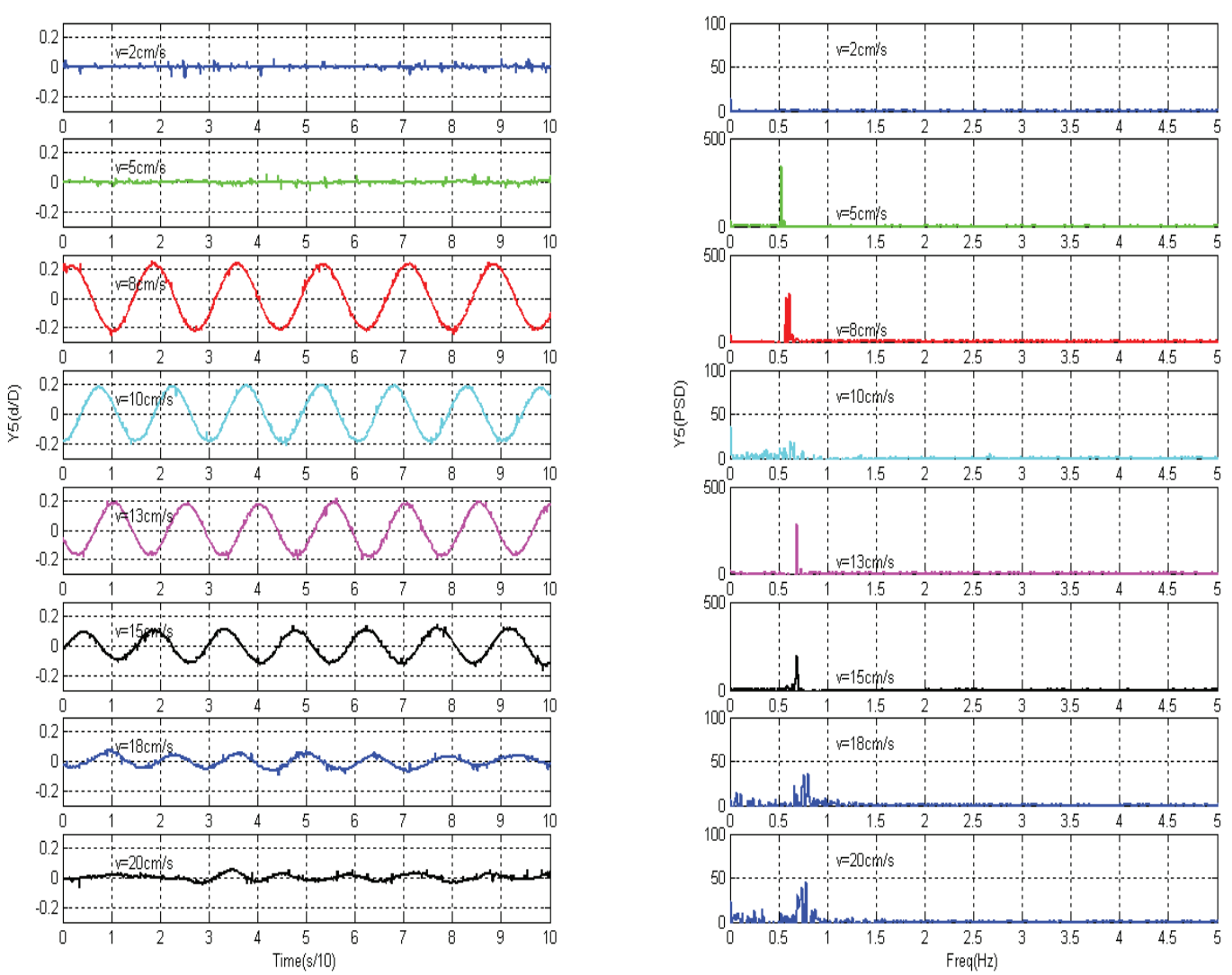

(a)

(b)

Figure 5. (a) Time histories of non-dimensional amplitudes of vertical oscillation and (b) their power spectral density (PSD) for $5^{\#}$ cylinder at different velocities. 


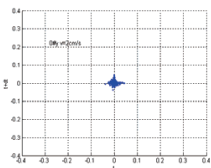

(a) $2 \mathrm{~cm} / \mathrm{s}$

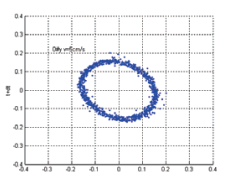

(b) $5 \mathrm{~cm} / \mathrm{s}$

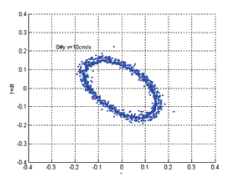

(c) $10 \mathrm{~cm} / \mathrm{s}$

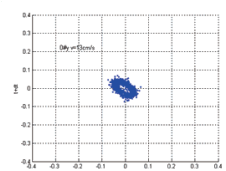

(d) $13 \mathrm{~cm} / \mathrm{s}$

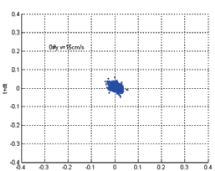

(e) $15 \mathrm{~cm} / \mathrm{s}$

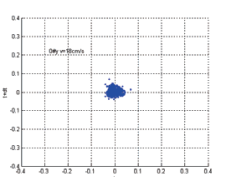

(f) $18 \mathrm{~cm} / \mathrm{s}$

Figure 6. Poincare sections of non-dimensional amplitude of vertical oscillation for $0^{\#}$ cylinder at different velocities.

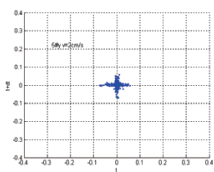

(a) $2 \mathrm{~cm} / \mathrm{s}$

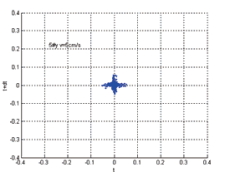

(b) $5 \mathrm{~cm} / \mathrm{s}$

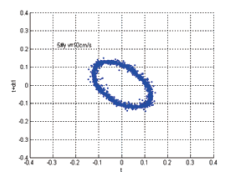

(c) $10 \mathrm{~cm} / \mathrm{s}$

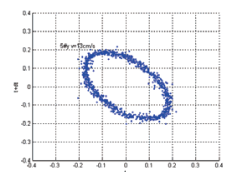

(d) $13 \mathrm{~cm} / \mathrm{s}$

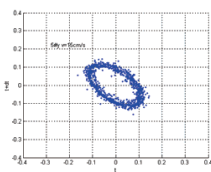

(e) $15 \mathrm{~cm} / \mathrm{s}$

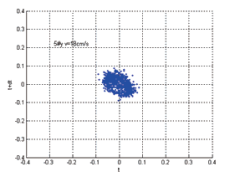

(f) $18 \mathrm{~cm} / \mathrm{s}$

Figure 7. Poincare sections of non-dimensional amplitude of vertical oscillation for $5^{\#}$ cylinder at different velocities.

(1) As shown in Fig.6 for experiments of cylinder $0^{\#}$, when the incoming velocity is increased from $2 \mathrm{~cm} / \mathrm{s}$ up to $20 \mathrm{~cm} / \mathrm{s}$, there are different features in Poincare sections. Points of signals are intensive and concentrative at velocity of $2 \mathrm{~cm} / \mathrm{s}$, indicating that the dynamic system is stable and cylinder oscillates weakly or almost holds still. As for velocity of 5 $\mathrm{cm} / \mathrm{s}$, projection of points shows a circular shape in Poincare section, which means the system getting into a periodic orbit and behavoring stablely although the oscillating amplitude is great. At velocity of $10 \mathrm{~cm} / \mathrm{s}$, such projection becomes elliptical, demonstrating that dynamic systerm enters into a new periodic orbit with weakened oscillation. While velocities are 15 and $18 \mathrm{~cm} / \mathrm{s}$, points in Poincare sections are concenstrated again associated with the system in stable state, as well as the oscillaing cylinder weaker or even still. Therefore, the incoming velocity with the occurrence of structural VIV is detected at $10 \mathrm{~cm} / \mathrm{s}$ quickly. In engineering application as for such structure, the flow velocity of $10 \mathrm{~cm} / \mathrm{s}$ should be avoided due to damage of structure by VIV.

(2) With same analyzing process from Fig.7 for cylinder $5^{\#}$, points of signals are concentrative with the stable system and nearly still cylinder at velocities of 2 and $5 \mathrm{~cm} / \mathrm{s}$. With velocities increasing up to 10,13 and $15 \mathrm{~cm} / \mathrm{s}$, the elliptic shape is appeared on Poincare section indicating that the system enters into a different periodic orbit. The amplitude of oscillation reaches up to the maximum at velocity of $13 \mathrm{~cm} / \mathrm{s}$. When velocity is $18 \mathrm{~cm} / \mathrm{s}$, points on Poincare section are concentrative and disperse, representing that the system behaviors at unstable state. At the meantime, the cylinder is oscillated weakly and non-periodically.

(3) Based on statistic analysis of signals, measured characteristic parameters, such as RMS values and timeaveraged values, are related with physical parameters, such as fluid velocity, frequency and oscillating amplitude. Hence, the actual velocity at which the synchronization of structure is occurred will be estimated from signals of one parameter. Then, the synchronization can be avoided early in engineering application.

\section{CONCLUSIONS}

1. By Poincare surface of sections, dynamic behaviors of manifold at two-dimensional, three-dimensional or highdimensional space, features of Poincare sections of manifold can be given qualitatively. The feature of such method is the investigation of high-dimensional system by lowering dimension.

2. Poincare section is a method used to quickly detect whether the structural VIV is occurred from time histories of measured signals. Experiments have shown that such method is quick and reliable as a new means of detection of VIV for engineering.

3. As for engineering application, the natural frequency of structure and vortex-shedding frequency should be estimated early as for different bodies. Different designed structures are applied in avoiding the occurrence of synchronization and further fatigue damage for specific ocean environment.

\section{REFERENCES}

[1] T. Sarpkya, "A critical review of the intrinsic nature of vortex-induced vibrations,” J. Fluids Struct., vol. 19, pp. 389-447, 2004.

[2] C.H.K. Williamson and R. Govardhan, "Vortex-induced vibrations," Annu. Rev. Fluid Mech., vol. 36, pp. 413-455, 2004.

[3] C.H.K. Williamson and R. Govardhan, "A brief review of recent results in vortex-induced vibrations," J. Wind Eng. Ind. Aerodyn., vol. 96, pp. 713-735, 2008.

[4] J.H. Kim and J. Stringer, Applied Chaos, A Wiley-Interscience Publication, John Wiley \& Sons, INC. 1997.

[5] A.A. Tsonis, Chaos form Theory to Application, Plenum Press, New York, 1992 